\title{
Status and Future Prospect of Muon Target at J- PARC MLF
}

\author{
Shunsuke Makimura ${ }^{1}$ \\ Muon Science Section, Materials and Life Science Division, J-PARC \\ Muon Science Laboratory, Institute of Materials Structure Science, High Energy Accelerator Research \\ Organization (IMSS-KEK) \\ 203-1, Shirakata, Tokai-Mura, Naka-gun, Ibaraki, 319-1106, Japan \\ E-mail: shunsuke.makimura@kek.jp
}

\section{Shiro Matoba}

Muon Science Section, Materials and Life Science Division, J-PARC

Muon Science Laboratory, IMSS-KEK

203-1, Shirakata, Tokai-Mura, Naka-gun, Ibaraki, 319-1106, Japan

E-mail: shiro.matoba@kek.jp

\section{Naritoshi Kawamura}

Muon Science Section, Materials and Life Science Division, J-PARC

Muon Science Laboratory, IMSS-KEK

203-1, Shirakata, Tokai-Mura, Naka-gun, Ibaraki, 319-1106, Japan

E-mail: nari.kawamura@kek.jp

\section{on behalf of Muon Science Section}

\begin{abstract}
A pulsed muon beam with unprecedented intensity will be generated by a $3 \mathrm{GeV} 333 \mu$ A proton beam on a muon target made of $20 \mathrm{~mm}$ thick isotropic graphite at J-PARC MLF MUSE (Muon Science Establishment). The first muon beam was successfully produced on September 26th, 2008. To extend the lifetime of the muon target, a muon rotating target, in which the radiation damage is distributed to a wider area, was installed in September of 2014. The rotating target has been successfully used for five years without replacement. Meanwhile, the failure on the rotation system that leads the possibility of the loss of the transmission was found during the longshutdown in September of 2018. The rotating target had been operated with the careful monitoring and the measures in preparation for the loss of the transmission from September 2018 to July 2019. In this report, the trouble of the rotation system and the measures at J-PARC MLF MUSE will be introduced.
\end{abstract}

The 21st international workshop on neutrinos from accelerators (NuFact2019)

August 26 - August 31, 2019

Daegu, Korea

\footnotetext{
${ }^{1}$ Speaker

(C) Copyright owned by the author(s) under the terms of the Creative Commons 


\section{Introduction}

A pulsed muon beam with unprecedented intensity will be generated by a $3 \mathrm{GeV} 333 \mu \mathrm{A}$ proton beam on a muon target made of $20 \mathrm{~mm}$ thick isotropic graphite at J-PARC MLF MUSE (Muon Science Establishment). The energy deposited by a $1 \mathrm{MW}$ proton beam is estimated to be $3.9 \mathrm{~kW}$ in the muon target. The first muon beam was successfully produced on September 26th, 2008. Gradually upgrading the beam intensity, continuous $300 \mathrm{~kW}$ proton beam operation has been conducted by a fixed target method without replacements till June of 2014 [1]. However, the lifetime of the fixed target will be less than 1 year by the proton-irradiation damage of graphite through $1 \mathrm{MW}$ proton beam operation. To extend the lifetime, a muon rotating target, in which the radiation damage is distributed to a wider area, had been developed [2]. The muon rotating target was installed in September of 2014. The rotating target has been successfully used for five years without replacement. Since 2018, continuous and stable $500 \mathrm{~kW}$ proton beam operation has been successfully conducted. Accumulated operational period and the number of revolutions reaches 17000 hours and 16 million in July, 2019. Furthermore, on 3rd, July of 2019, 1 MW beam operation for 11 hours (Beam power, $935 \mathrm{~kW}$ : $1 \mathrm{MW}$ equivalent for one pulse) was successfully achieved. Meanwhile, the damage on the flexible coupling in the rotation system was found during the long-shutdown in September of 2018. The rotating target had been operated with the careful monitoring and the measures in preparation for the loss of the transmission from September 2018 to July 2019. In this report, the trouble of the rotation system and the measures at J-PARC MLF MUSE will be introduced.

\section{Failure on the rotation system}

When the rotation feedthrough between a vacuum and air was replaced as a regular maintenance in September, 2018, the damage on the flexible coupling that connects the feedthrough and the rotation shaft was found. Though the coupling had survived in spite of the damage, it completely broke to pieces through the replacement process. We recognized that the damage originates from the drawing instructions for the machining of the coupling. The inappropriate couplings were employed on the two positions of the rotation system. Figure 1 shows pictures of the rotating target assembly (right), the replacement of the vacuum feed-through with the glove box (left-up), and the broken flexible coupling (left-down). While the broken coupling was replaced with the appropriate one immediately, it was difficult for another one to replace before the restart of the beam operation because of its uneasy

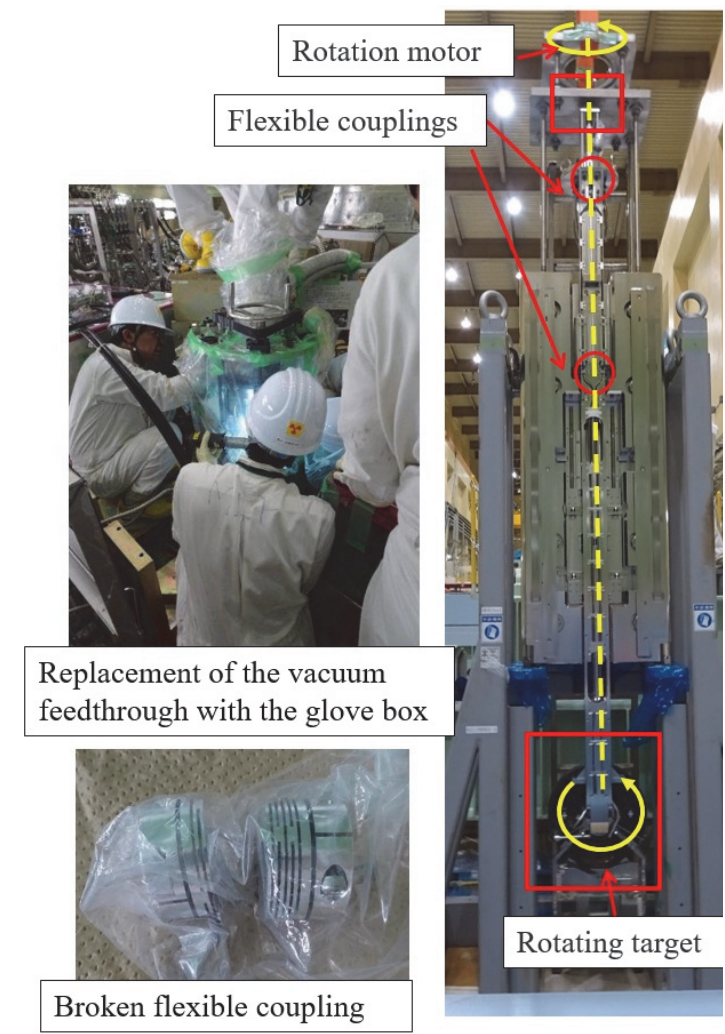

Figure 1: Pictures of the rotating target assembly (Right), the replacement of the vacuum feed-through with the glove box (left-up), and the broken flexible coupling (left-down) 
accessibility, the high residual radiation dose, and the high contamination of tritium. Consequently, we decided to keep on utilizing the inappropriate coupling with the careful monitoring and the measures in preparation for the loss of the transmission.

\section{Analysis of rotation stop}

The rotation stops during the proton beam operation had to be detected the most reliably by the increment of the torque of the rotation motor. When the coupling breaks down, the detection might not work as expected. Therefore, the variation of the temperature and the subsequent thermal stress were carefully evaluated through a finite element method, ANSYS [3].

The graphite itself is divided to three pieces to release thermal stress originating the temperature difference on the graphite. The divided three pieces are unified as the target wheel by the centrifugal-force ring made of Nickel-based alloy. The weight of the target wheel is $3 \mathrm{~kg}$, and the temperature of the target wheel reaches $930 \mathrm{~K}$. The target wheel is supported by the rigid wheel support with the nine flat bars made of nickel-based alloy. The cutting section of the flat bar is rectangular of $1 \mathrm{~mm}$ by $10 \mathrm{~mm}$, and the length of the bar is $35 \mathrm{~mm}$. The rectangle is located in parallel with circumferential direction of the target wheel to release the thermal stress originating the large temperature difference between the target wheel and the wheel support as well as to support the weight of the target wheel. Figure 2 shows a picture of the rotating target. To validate the effect of the unexpected rotation stop, the maximum temperature of the graphite, the thermal stress on the graphite, the thermal stress on the flat bar are evaluated. To save time for the simulation, the simplified 3-dimensional model of the graphite wheel is used for FEM code since the results are almost similar to the full model. The thermal stress on the flat bar is evaluated by a theoretical calculation with a double supported beam model under the condition of the rotation is restricted on the both ends. The criteria of the maximum temperature of the graphite, the thermal stress on the graphite, the thermal stress on the flat bar are $2300 \mathrm{~K}, 25 \mathrm{MPa}, 200 \mathrm{MPa}$, respectively.

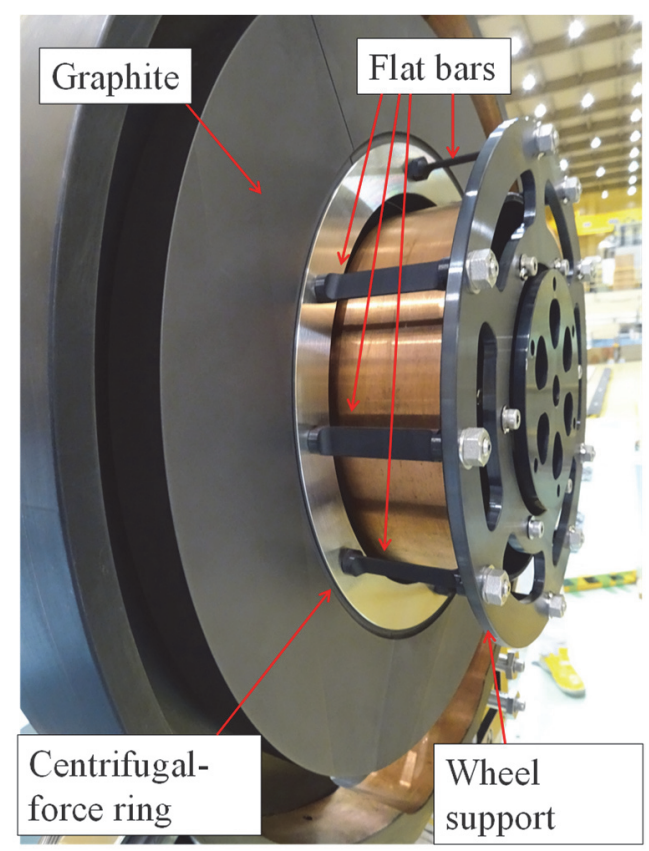

Figure 2: A picture of the rotating target

Table 1 shows the results of the analyses under the conditions of $500 \mathrm{~kW}$ and $1000 \mathrm{~kW}$ with rotation (W/R) and the conditions of $500 \mathrm{~kW}, 700 \mathrm{~kW}$, and $1000 \mathrm{~kW}$ without rotation (W/O/R). As the results of simulations, the allowable beam intensity without rotation is proved to be 500 $\mathrm{kW}$. When the beam intensity is beyond $500 \mathrm{~kW}$ without rotation, the graphite is probably cracked and the flat bar is plasticly deformed. However, even if the temperature of the graphite reaches to $2300 \mathrm{~K}$, the evaporation rate of the graphite is still low enough $(1.4 \mu \mathrm{m} /$ hour $)$. Furthermore, the used rotating target can be replaced with a new one, because the aperture of the target chamber is large enough to extract the rotating target, even composed of the plasticly-deformed flat bars. On the other hand, the radioactive gases including tritium are produced and stored in the graphite 
during the beam operation. The radioactive gases from the graphite at high temperature should be taken into account.

Table 1: Results of the analyses under the conditions of $500 \mathrm{~kW}$ and $1000 \mathrm{~kW}$ with rotation $(\mathrm{W} / \mathrm{R})$ and the conditions of $500 \mathrm{~kW}, 700 \mathrm{~kW}$, and $1000 \mathrm{~kW}$ without rotation (W/O/R).

\begin{tabular}{|c|c|c|c|c|c|c|}
\hline & Criteria & $\begin{array}{l}500 \mathrm{~kW} \\
\mathrm{~W} / \mathrm{R}\end{array}$ & $\begin{array}{l}1000 \mathrm{~kW} \\
\mathrm{~W} / \mathrm{R}\end{array}$ & $\begin{array}{l}500 \mathrm{~kW} \\
\mathrm{~W} / \mathrm{O} / \mathrm{R}\end{array}$ & $\begin{array}{l}700 \mathrm{~kW} \\
\text { W/O/R }\end{array}$ & $\begin{array}{l}1000 \mathrm{~kW} \\
\text { W/O/R }\end{array}$ \\
\hline Max. temperature of Graphite (G.) (K) & 2300 & 740 & 880 & 1690 & 1990 & 2390 \\
\hline Temperature difference on Graphite (K) & - & 30 & 50 & 950 & 1220 & 1600 \\
\hline Equivalent stress on Graphite (MPa) & 25 & 0.7 & 1.1 & 22 & 29 & 38 \\
\hline Temperature of CF ring & - & - & 450 & 910 & 1000 & - \\
\hline Equivalent stress on flat bar & 200 & - & 80 & 180 & 240 & - \\
\hline
\end{tabular}

\section{Restart of beam operation}

Because the results showed the $500 \mathrm{~kW}$ of the beam operation is acceptable, we decided to keep on utilizing the current rotating target with the inappropriate couplings. To detect the rotation stop immediately, the sensitivity of the machine protection system, such as the quadrupole mass spectrometer, the torque monitor of the rotation system, and the temperature measurement has been improved. To avoid the release of the emitted radioactive gases to the outside of the facility via the vacuum pumping system, the buffer tank was implemented to keep the pumped gas tentatively until their activities are measured and are confirmed to be low enough. Figure 3 demonstrates a schematic view of the buffer tank system. The rotating target had retreated to the escape position from 20th October to 6th November to validate the new vacuum pumping system and the buffer tank. After resume of the rotating target, the $500 \mathrm{~kW}$ proton beam operation had been stably conducted. In the long-shutdown of 2019, the replacement of the entire rotating target

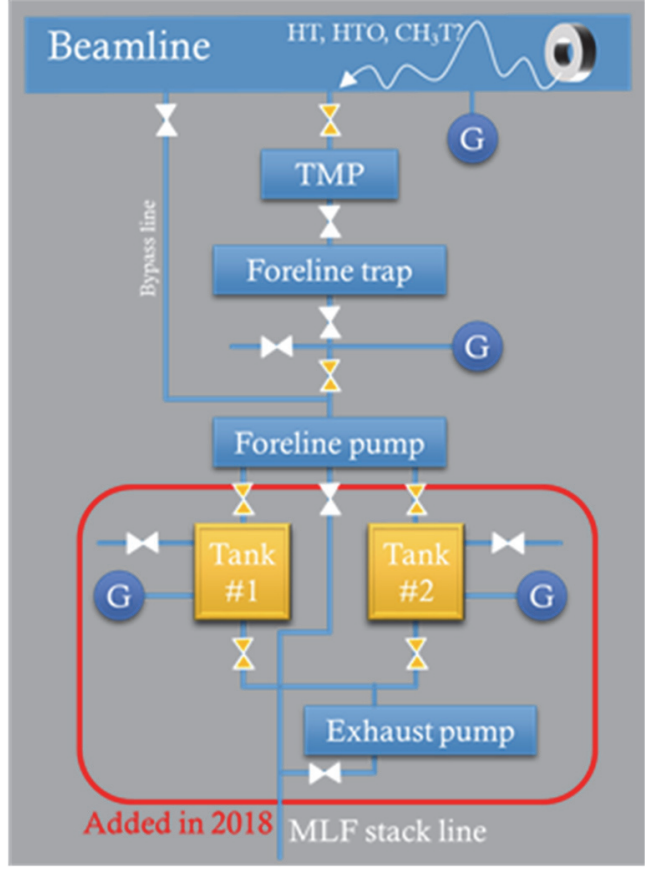

Fig. 3: A schematic view of the buffer tank system. assembly was successfully completed.

\section{Future Prospect of Muon Target at J-PARC MLF}

The temperature of the muon rotating target cannot be measured by direct contact because it is not possible for a thermometer thermocouples to be attached to the rotating body. Thermocouples are attached to the cooling jacket to measure the temperature rise due to thermal radiation from the muon target. However, in the case of a significant temperature rise, due to the delay in the thermocouple detection, this monitoring system cannot stop the proton beam quickly. To realize the quick measurements we are developing a real-time two-dimensional radiation 
thermometer which can monitor the temperature of muon rotating target indirectly. The thermometer will be installed in September of 2019, and a validation run will initiate.

\section{Acknowledgement}

I would like to thank to support of the dedicated staffs from The NIPPON ADVANCED TECHNOLOGY CO., LTD, Japan [4].

\section{References}

[1] S. Makimura et al., JPS Conf. Proc. , 051002 (2015), http://dx.doi.org/10.7566/JPSCP.8.051002.

[2] S. Makimura et al., Journal of Radioanalytical and Nuclear Chemistry 305 (2015) 811-815.

[3] https://www.ansys.com/, accessed in November 2019.

[4] https://nat-web.com/, written in only Japanese, and accessed in November 2019. 